\title{
MRI-Visible Perivascular Spaces in the Centrum Semiovale Are Associated with Brain Amyloid Deposition in Patients with Alzheimer Disease-Related Cognitive Impairment
}

\author{
(D)H.J. Kim, (D). Cho, (D) M. Park, DJ.W. Kim, (D) S.J. Ahn, (D) C.H. Lyoo, DS.H. Suh, and (D).H. Ryu
}

\section{ABSTRACT}

BACKGROUND AND PURPOSE: The association of perivascular spaces in the centrum semiovale with amyloid accumulation among patients with Alzheimer disease-related cognitive impairment is unknown. We evaluated this association in patients with Alzheimer disease-related cognitive impairment and $\beta$-amyloid deposition, assessed with $\left[{ }^{18} \mathrm{~F}\right]$ florbetaben PET/CT.

MATERIALS AND METHODS: MR imaging and $\left[{ }^{18} \mathrm{~F}\right]$ florbetaben PET/CT images of 144 patients with Alzheimer disease-related cognitive impairment were retrospectively evaluated. MR imaging-visible perivascular spaces were rated on a 4-point visual scale: a score of $\geq 3$ or $<3$ indicated a high or low degree of MR imaging-visible perivascular spaces, respectively. Amyloid deposition was evaluated using the brain $\beta$-amyloid plaque load scoring system.

RESULTS: Compared with patients negative for $\beta$-amyloid, those positive for it were older and more likely to have lower cognitive function, a diagnosis of Alzheimer disease, white matter hyperintensity, the Apolipoprotein E $\varepsilon 4$ allele, and a high degree of MR imaging-visible perivascular spaces in the centrum semiovale. Multivariable analysis, adjusted for age and Apolipoprotein E status, revealed that a high degree of MR imaging-visible perivascular spaces in the centrum semiovale was independently associated with $\beta$-amyloid positivity (odds ratio, 2.307; 95\% CI, 1.036-5.136; $P=.041$ ).

CONCLUSIONS: A high degree of MR imaging-visible perivascular spaces in the centrum semiovale independently predicted $\beta$-amyloid positivity in patients with Alzheimer disease-related cognitive impairment. Thus, MR imaging-visible perivascular spaces in the centrum semiovale are associated with amyloid pathology of the brain and could be an indirect imaging marker of amyloid burden in patients with Alzheimer disease-related cognitive impairment.

ABBREVIATIONS: $\mathrm{AD}=$ Alzheimer disease; $\mathrm{ADCl}=\mathrm{AD}$-related cognitive impairment; $\mathrm{APOE}=$ Apolipoprotein $\mathrm{E} ; \mathrm{BAPL}=\beta$-amyloid plaque load; $\left[{ }^{18} \mathrm{~F}\right] \mathrm{FBB}$ $=\left[{ }^{18} \mathrm{~F}\right]$ florbetaben; MMSE $=$ Mini-Mental State Examination; $\mathrm{PVS}=$ perivascular spaces; PVS-CS $=$ perivascular spaces in the centrum semiovale; SUVr $=$ standardized uptake value ratios; $\mathrm{WMH}=$ white matter hyperintensity

$A$ ccumulating evidence suggests that MR imaging-visible perivascular spaces (PVS) are not innocent lesions but may be a neuroimaging marker of cerebral small-vessel disease. ${ }^{1-3}$ The

Received June 18, 2020; accepted after revision January 21, 2021.

From the Departments of Nuclear Medicine (H.J.K., Y.H.R.), Neurology (H.C., C.H.L.), and Radiology (M.P., J.W.K., S.J.A., S.H.S.), Gangnam Severance Hospital, Yonsei University College of Medicine, Seoul, South Korea; and Department of Nuclear Medicine (H.J.K.), Yongin Severance Hospital, Yonsei University College of Medicine, Yongin-si, South Korea.

H.J. Kim and H. Cho contributed equally to this work.

This research was supported by the Basic Science Research Program through the National Research Foundation of Korea funded by the Ministry of Education, Science and Technology (NRF-2017RIDIA1B03034388), the National Research Foundation of Korea grant funded by the Korean government (Ministry of Science and ICT) (No. NRF-2020RIC1C1005724), and Yonsei University College of Medicine (6-2019-0059).

Please address correspondence to Mina Park, MD, Department of Radiology, Gangnam Severance Hospital, Yonsei University College of Medicine, Eonjuro 211, Gangnam-gu, Seoul, South Korea; e-mail: to.minapark@yuhs.ac

- Indicates open access to non-subscribers at www.ajnr.org

Indicates article with online supplemental data.

http://dx.doi.org/10.3174/ajnr.A7155 perivascular space is a potential space filled with interstitial fluid surrounding penetrating vessels. It is involved in the drainage of interstitial fluid and solutes from the brain. ${ }^{4}$ Therefore, several clinical conditions that reduce the clearance of solutes from the brain interstitial fluid such as aging, hypertension, and inflammation can result in MR imaging-visible PVS. ${ }^{5}$ MR imaging-visible PVS are also associated with various diseases, such as traumatic brain injury, Parkinson disease, and dementia. ${ }^{6-9}$ The location of MR imaging-visible PVS is an important factor to consider when predicting disease status because MR imaging-visible PVS in the basal ganglia may be associated with markers of arteriolosclerosis, whereas MR imaging-visible PVS in the centrum semiovale (PVSCS) are linked to diseases involving amyloid pathology, such as Alzheimer disease (AD) and cerebral amyloid angiopathy. ${ }^{10,11}$

Many different studies on cerebral amyloid angiopathy have demonstrated a strong relationship between MR imaging-visible PVS-CS and cerebral amyloid angiopathy. ${ }^{12-15}$ Some studies have 


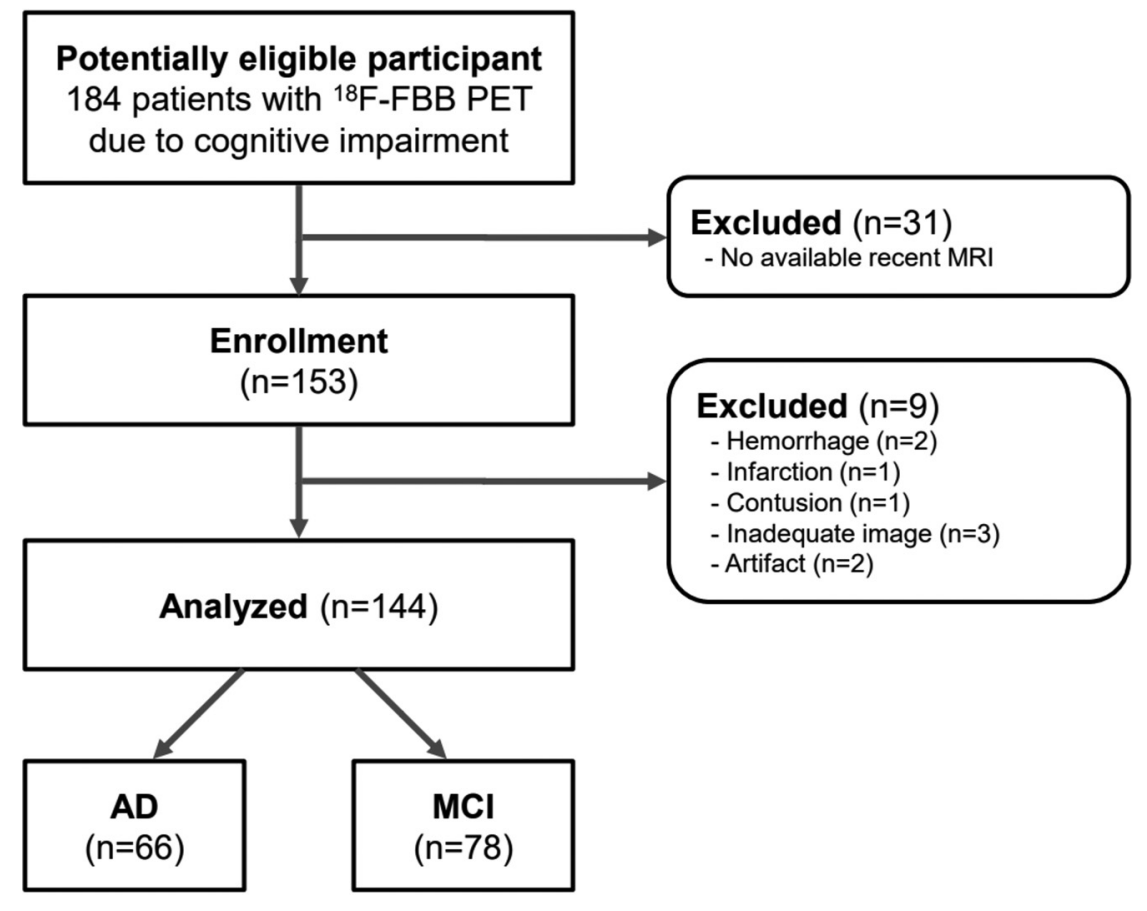

FIG 1. Patient-inclusion flowchart.

that labels in vivo amyloid deposits, in patients with cognitive impairment.

\section{MATERIALS AND METHODS \\ Participants}

The need for written informed consent from patients was waived by the institutional review board of Gangnam Severance Hospital due to the retrospective nature of this study. Data were reviewed from 153 consecutive patients with cognitive impairment and clinical indications of $\mathrm{AD}$-related cognitive impairment (ADCI). All patients underwent an $\left[{ }^{18} \mathrm{~F}\right] \mathrm{FBB}$ PET/CT and brain MR imaging within a 3-month interval from June 2017 to July 2019. Of the 153 patients with ADCI, we excluded 3 patients with inadequate image acquisition, 2 with image artifacts, 2 with intracranial hemorrhage, 1 with a large territorial infarction, and 1 with an old traumatic contusion. Therefore, 144 patients with ADCI were finally included in the analysis; among them, 66 patients had probable $\mathrm{AD}$ and 78

suggested that the dilation of PVS and failure in the drainage of interstitial fluid may result from deposition of $\beta$-amyloid in the cortical and leptomeningeal arteries. ${ }^{16}$ Furthermore, evidence indicates that MR imaging-visible PVS-CS are associated with in vivo $\beta$-amyloid deposition in the brain, based on amyloid PET scanning, ${ }^{14,17}$ which enables the visualization of brain amyloid deposition and measures the distribution and density of $\beta$-amyloid plaques. ${ }^{18}$

Failure in the perivascular clearance of $\beta$-amyloid may also be involved in the accumulation of $\beta$-amyloid in $\mathrm{AD} .{ }^{19}$ In patients with $\mathrm{AD}, \mathrm{MR}$ imaging-visible PVS-CS may reflect impaired perivascular clearance of $\beta$-amyloid, and several studies have indicated a link between MR imaging-visible PVS and AD.,20 However, unlike evidence for the association between MR imaging-visible PVS-CS and cerebral amyloid angiopathy, scant evidence exists regarding the association between $\beta$-amyloid deposition and MR imaging-visible PVS in the population with dementia.

Several compounds labeled with radioisotopes have been developed to image amyloid deposition. In patients with cognitive impairment, PET scans using these tracers are widely used for diagnosis and follow-up. ${ }^{21}$ Among the radiopharmaceuticals, $\left[{ }^{18} \mathrm{~F}\right]$ florbetaben $\left(\left[{ }^{18} \mathrm{~F}\right] \mathrm{FBB}\right)$ is widely used for PET imaging to evaluate $\mathrm{AD}$ and other causes of dementia. $\left[{ }^{18} \mathrm{~F}\right] \mathrm{FBB}$ has a proper half-life and also allows high-resolution image acquisition, diagnostic capability, and quantification. ${ }^{22}$ For these reasons, $\left[{ }^{18} \mathrm{~F}\right]$ FBB is suitable for evaluating amyloid accumulation and its association with enlarged PVS in patients with dementia.

We hypothesized that MR imaging-visible PVS-CS would be associated with brain amyloid deposition in cognitively impaired patients, as it is in patients with cerebral amyloid angiopathy. We also evaluated the association using $\left[{ }^{18} \mathrm{~F}\right] \mathrm{FBB}$, a PET radiotracer had mild cognitive impairment. Figure 1 shows the patient-inclusion flowchart. The criteria for probable $\mathrm{AD}$, proposed by the National Institutes of Neurological and Disorders and Stroke and by the Alzheimer's Disease and Related Disorders Association ${ }^{23}$, and the Petersen criteria, ${ }^{24}$ were used for the clinical diagnosis of mild cognitive impairment.

\section{Clinical Evaluation}

We assessed all available patient information, such as basic demographic characteristics, other medical conditions (including a history of vascular risk factors), global cognitive assessment scores (eg, Clinical Dementia Rating Scale-Sum of Boxes score, MiniMental State Examination [MMSE] score, and a standardized neuropsychological battery called the Seoul Neuropsychological Screening Battery ${ }^{25}$ ), and Apolipoprotein E (APOE) $\varepsilon 4$ genotyping. $A P O E$ genotyping was performed using the polymerase chain reaction. Individuals with at least $1 \varepsilon 4$ allele were classified as APOE $\varepsilon$ 4-positive.

\section{MR Imaging Acquisition and Analysis}

The MR imaging sequences were performed on a $3 \mathrm{~T}$ scanner (Discovery MR750; GE Healthcare) with a 16-channel head coil. All patients underwent axial T2-weighted imaging, sagittal T1weighted imaging, sagittal 3D-FLAIR, and axial 3D susceptibilityweighted angiography. Axial 2D T2-weighted images were acquired using the FSE sequence (TR/TE, 5320/102 ms; flip angle, $142^{\circ}$; section thickness, $4 \mathrm{~mm}$; gap, $1 \mathrm{~mm}$; FOV, $230 \mathrm{~mm}$; matrix, $352 \times 352)$. The actual TR/TE ranged from $5289 / 104 \mathrm{~ms}$ to $6028 /$ $97 \mathrm{~ms}$ due to the autoTR setting and specific absorption rate adjustment. Sagittal 3D T1-weighted images were obtained using the $3 \mathrm{D}$ fast-spoiled gradient echo sequence (TR/TE, 8.2/3.2 ms; 
flip angle, $12^{\circ}$; section thickness, $1 \mathrm{~mm}$; FOV, $240 \mathrm{~mm}$; matrix, $256 \times 256)$. Sagittal 3D-FLAIR images were obtained using the Cube sequence (GE Healthcare) (TR/TE, 6000/89 ms; TI, $1741 \mathrm{~ms}$; section thickness, $1.2 \mathrm{~mm}$; FOV, $260 \mathrm{~mm}$; matrix, $256 \times 224)$. Axial 3D susceptibility-weighted angiography images were obtained using the following parameters: TR/TE, 30.9/ $23.4 \mathrm{~ms}, 46.8 \mathrm{~ms}$, and $70.2 \mathrm{~ms}$; flip angle, $10^{\circ}$; section thickness, $2 \mathrm{~mm}$; gap, $1 \mathrm{~mm}$; FOV, $230 \mathrm{~mm}$; and matrix, $320 \times 224$.

The PVS that were visible on MR imaging were assessed in line with the STandards for ReportIng Vascular changes on nEuroimaging recommendations. ${ }^{26}$ Based on the axial T2weighted MR images, MR imaging-visible PVS were rated in the basal ganglia and centrum semiovale using a validated 4-point visual rating scale: $0=$ no PVS; $1=\leq 10$ PVS; $2=11-20$ PVS; $3=21-40$ PVS; and $4=\geq 40$ PVS. $^{12,27}$ The numbers refer to MR imaging-visible PVS on 1 side of the brain (ie, the side/section with the highest number of PVS after all relevant slices for each anatomic area were reviewed). We prespecified a dichotomized classification of MR imaging-visible perivascular space degree as "high degree" (ie, score of $>2$ ) or "low degree" (ie, score of $\leq 2$ ). This definition is in line with the perivascular space burden used in previous studies and may be characteristic of amyloid pathology. ${ }^{10,12}$

White matter hyperintensities (WMHs) were defined as hyperintense white matter lesions on FLAIR images based on the STandards for ReportIng Vascular changes on nEuroimaging criteria and were graded using the Fazekas scale as "deep WMHs" ( 0 = absent; 1 = punctate; $2=$ early confluent; $3=$ confluent $)$ or "periventricular WMHs" ( $0=$ absent; $1=$ caps or pencil-thin lining; $2=$ smooth halo; $3=$ irregular WMHs extending into the deep white matter). ${ }^{26,28}$ The total Fazekas score was calculated by adding the periventricular and deep WMH scores. A score of $>3$ was considered WMH-positive. ${ }^{28}$ Lacunes were defined as small lesions that were hypointense on T1-weighted images and hyperintense on T2-weighted images and had perilesional halos on FLAIR images. ${ }^{26}$ Microbleeds were defined as small signal voids with associated blooming on susceptibility-weighted angiography images. The presence and number of lacunes and microbleeds were recorded as previously described. ${ }^{26}$

\section{$\left[{ }^{18}\right.$ F] FBB PET Imaging Acquisition and Analysis}

PET images were obtained using a Biograph mCT PET/CT scanner (Siemens). At 90 minutes after we injected 307.0 (SD, 32.2) $\mathrm{MBq}$ of $\left[{ }^{18} \mathrm{~F}\right] \mathrm{FBB}, \mathrm{PET}$ data were acquired for 20 minutes. After we conducted attenuation and scatter correction, 3D-PET images were reconstructed in a $256 \times 256 \times 223$ matrix with a voxel size of $1.591 \times 1.591 \times 1 \mathrm{~mm}$ using the ordered-subsets expectation maximization algorithm.

We defined the results of amyloid PET as "positive" when the visual assessment of $\left[{ }^{18} \mathrm{~F}\right]$ FBB PET was scored as 2 or 3 on the brain $\beta$-amyloid plaque load (BAPL) scoring system based on the following: $1=$ no tracer uptake, $2=$ moderate tracer uptake, and $3=$ pronounced tracer uptake. ${ }^{29,30}$ The decision was based on visual assessment of each section on the axial plane. All scans were independently evaluated by 2 experienced nuclear medicine physicians, who reread all the studies while blinded to the original clinical reports and clinical information and reached a consensus.
In addition to the visual assessment, we also performed a semi-quantitative analysis to evaluate the cortical $\left[{ }^{18} \mathrm{~F}\right] \mathrm{FBB}$ retention in the PET/CT scans, as follows: Cortical regional standardized uptake value ratios (SUVr) were calculated for each patient in the 6 cortical ROIs (frontal, parietal, lateral temporal, precuneus, and anterior and posterior cingulate cortex regions). We used the cerebellar gray matter as the reference for SUVr calculation. The global composite florbetaben SUVr was calculated as the average of the SUVr value in each ROI. ${ }^{29,31}$ On the basis of the SUVr analysis, an $\left[{ }^{18} \mathrm{~F}\right] \mathrm{FBB}$ PET was defined as positive (SUVr-positive) when the global composite florbetaben SUVr was $>1.42$, which was assessed against the histopathologic determination of $\beta$-amyloid in previous research. ${ }^{32}$

\section{Statistical Analyses}

Baseline characteristics were compared using the $\chi^{2}$ or Fisher exact test for categoric variables, independent $t$ tests for normally distributed continuous variables, and Mann-Whitney $U$ tests for continuous variables that were not normally distributed. MR imaging-visible PVS in both the basal ganglia and centrum semiovale were considered categoric variables, respectively. They were subdivided by severity, as described previously. We explored the independent and pathophysiologically relevant predictors of brain amyloid deposition using logistic regression analyses based on our prespecified hypothesis and the results of univariable analyses (including variables with $P<.05$ ). Multivariable logistic regression analyses, including age, sex, $A P O E \varepsilon 4$ allele status, and high degree of MR imaging-visible PVS-CS were performed. The variables of interest in univariable analysis were included in the multivariable models using the enter method. Positive WMH was not included in the analysis because it was significantly associated with a high degree of MR imaging-visible PVS-CS $\left(P<.001\right.$, based on the $\chi^{2}$ test $)$.

\section{Random Forests Analysis}

A total of 13 demographic and radiologic features, excluding $\mathrm{WMH}$, were evaluated; these features included age, sex, hypertension, diabetes, hyperlipidemia, previous stroke, APOE $\varepsilon 4$ allele, MR imaging-visible PVS in the basal ganglia, MR imaging-visible PVS-CS, lacunes, cortical superficial siderosis, lobar cerebral microbleeds, and deep cerebral microbleeds. The random forests model was trained with demographic and radiologic features to classify the amyloid positivity of the brain. The diagnostic ability of the random forests model using receiver operating characteristic analysis and the area under the receiver operating characteristic curve was calculated.

\section{RESULTS}

\section{Study Participants}

In this study, the total number of patients with ADCI was 144, comprising 67 patients with a BAPL score of one, 11 with a BAPL score of 2 , and 66 with a BAPL score of 3 . On the basis of the criteria of the BAPL scoring system, 67 patients were negative for $\beta$-amyloid deposition and 77 were positive for it. According to the SUVr analysis, 74 patients were negative for $\beta$-amyloid deposition and 70 patients were positive for it. 
Among the 144 patients with ADCI, 3 had a PVS in the basal ganglia score of zero, 85 had a score of 1 in MR imaging-visible PVS in the basal ganglia, 32 had a score of two, 17 had a score of 3 , and 7 had a score of 4 in terms of MR imaging-visible PVS in the basal ganglia. With regard to MR imaging-visible PVS-CS, 15 patients with ADCI had a score of one, 57 had a score of two, 56 had a score of 3 , and 16 had a score of 4 .

\section{Comparison between Groups Positive and Negative for $\beta$-Amyloid}

Age was significantly older in the patients positive for $\beta$-amyloid deposition than in patients negative for it (mean, 75.4 [SD,
7.6] years versus $71.3[\mathrm{SD}, 10.6]$ years; $P=.010)$. The prevalence of the APOE $\varepsilon 4$ allele $(P=.001)$, WMH $(P=.013)$, and $\mathrm{AD}$ $(P<.001)$ was higher in patients with $\beta$-amyloid positivity than in patients with $\beta$-amyloid negativity. The patients with $\beta$-amyloid positivity had poorer cognitive function on the MMSE $(P<.001)$, the Clinical Dementia Rating Scale $(P=.019)$, and the Clinical Dementia Rating Scale-Sum of Boxes $(P<.001)$ compared with patients with $\beta$-amyloid negativity (Table 1 ). A high degree of MR imaging-visible PVS-CS existed more frequently among patients with $\beta$-amyloid positivity than in patients with $\beta$-amyloid negativity (48/77 [62.3\%] versus 24/67 [35.8\%]; $P=.002$ ), whereas a high degree of MR imaging-visible PVS in the basal ganglia did not differ between groups positive and negative for $\beta$-amyloid (13/77 [16.9\%] versus 11/67 [16.4\%], $P=.297$ ) (Fig 2). Representative examples of PVS patterns with the corresponding $\left[{ }^{18} \mathrm{~F}\right] \mathrm{FBB}$ PET findings are presented in Fig 3.

\section{Quantitative SUVr Analysis}

In the SUVr analysis, 43/70 (61.4\%) with global composite SUVr positivity were classified as having a high degree of MR imaging-visible PVS-CS compared with 29/74 (39.2\%) with SUVr negativity $(P=.008)$, and the high degree of MR imaging-visible PVS in the basal ganglia did not differ between the SUVr-positive and SUVrnegative groups (12/70 [17.1\%] versus 12/74 [16.2\%], $P=.881)$. The global composite SUVr was significantly higher in patients with a high degree of MR imaging-visible PVS-CS than in those with a low degree (1.52 versus $1.37, P=.005)$. In region-based analysis, all 6 ROIs showed statistically significant differences. The frontal (1.56 versus 1.37, $P=.004)$, parietal (1.50 versus $1.38, P=.009)$, lateral temporal (1.31 versus $1.19, P=.008)$, precuneus (1.60 versus $1.43, P=.008)$, anterior cingulate ( 1.47 versus $1.36, P=.044)$, and posterior cingulate (1.69 versus $1.52, P=.004)$ regions showed higher SUVr values in the patients with a high degree of MR imaging-visible PVS-CS than in those a low degree, respectively.

\section{MR Imaging-Visible PVS as a Predictor of $\beta$-Amyloid Positivity}

In the univariate logistic regression analysis, a high degree of MR imaging-visible PVS-CS was a positive predictor of $\beta$-amyloid positivity based 
on the BAPL scoring system (OR, 2.966; 95\% CI, 1.503-5.851; $P=.002$ ) (Table 2). After adjustment, a high degree of MR imaging-visible PVS-CS remained independently associated with $\beta$-amyloid PET positivity (OR, 2.307; 95\% CI, 1.036-5.136; $P=.041$ ), as well as the presence of the APOE $\varepsilon 4$ allele (OR, 4.583; 95\% CI, 1.945-10.796; $P<.001)$ and older age (OR, 1.050; 95\% CI, 1.004-1.098; $P=.034)$.

The random forests model with 13 variables showed an area under the curve of 0.985 (95\% CI, 0.964-1.000) with an accuracy of 0.971 (95\% CI, 0.931-1.000), sensitivity of 1.000 (95\% CI, 1.000-

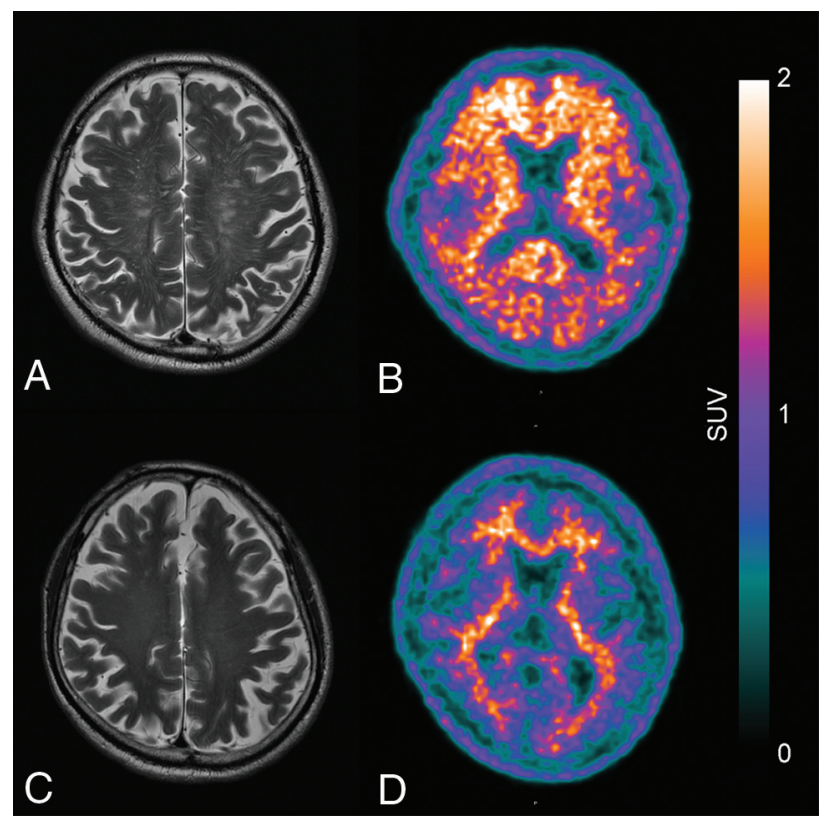

FIG 3. Examples of perivascular space patterns with the corresponding $\left[{ }^{8} \mathrm{~F}\right]$ FBB PET image. The axial T2-weighted MR imaging shows a high degree of MR imaging-visible PVS-CS $(A)$, and the corresponding $\left[{ }^{18} \mathrm{~F}\right]$ FBB PET (B) shows pronounced $\beta$-amyloid deposition. Axial T2weighted MR imaging shows a low degree of MR imaging-visible PVS$\mathrm{CS}(C)$ and the $\left[{ }^{18} \mathrm{~F}\right]$ FBB PET scan $(D)$ shows low $\beta$-amyloid deposition.
1.000), and specificity of 0.985 (95\% CI, 0.964-1.000). We observed that MR imaging-visible PVS-CS ranked as the third most important variable after APOE 4 and age (Online Supplemental Data).

\section{Comparisons between Patients with High and Low Degrees of MR Imaging-Visible PVS-CS among Patients Positive for $\boldsymbol{\beta}$-Amyloid}

Among the 77 patients with ADCI with $\beta$-amyloid positivity, $70.8 \%$ of the patients with a high degree of MR imaging-visible PVS-CS had a higher proportion of positive WMHs (34/48), whereas $44.8 \%(13 / 29)$ of patients with a low degree of MR imaging-visible PVS-CS had positive WMHs $(P=.023)$. The frequency of lobar microbleeds was also higher in patients with ADCI and $\beta$-amyloid positivity than among patients with ADCI and $\beta$-amyloid negativity $(P=.007)$. Other parameters such as age, MMSE score, lacunes, deep microbleeds, sex, hypertension, diabetes mellitus, hyperlipidemia, previous stroke, the presence of an $A P O E \varepsilon 4$ allele, or final diagnosis were not significantly different between the patients with high and low degrees of MR imaging-visible PVS-CS (Online Supplemental Data).

\section{Comparisons between Patients with High and Low Degrees of MR Imaging-Visible PVS-CS among Patients Negative for $\beta$-Amyloid}

Among 67 patients with ADCI with $\beta$-amyloid negativity, the patients with a high degree of MR imaging-visible PVS-CS ( $n=24)$ were older compared with patients with a low degree of MR imaging-visible PVS-CS $(n=43)$ (mean, 76.5 [SD, 9.8] years versus $68.3[\mathrm{SD}, 10.0]$ years; $P=.002$ ). Furthermore, the patients with a high degree of MR imaging-visible PVS-CS had lower MMSE scores compared with those with a low degree of MR imaging-visible PVS-CS (23.8 [SD, 4.0] versus 26.1 [SD, 3.7], $P=.018$ ). The patients with a high degree of MR imaging-visible PVS-CS compared with patients with a low degree of MR imaging-visible PVS-CS had a higher prevalence of hypertension (54.2\% versus $27.9 \%, P=.033$ ), positive WMHs (58.3\% versus $30.2 \%, P=.025)$, final diagnosis of probable $\mathrm{AD}(45.8 \%$ versus

Table 2: Logistic regression analysis for the predictors of $\beta$-amyloid positivity

\begin{tabular}{|c|c|c|c|c|}
\hline & \multicolumn{2}{|c|}{ Univariable } & \multicolumn{2}{|c|}{ Multivariable } \\
\hline & OR $(95 \% \mathrm{Cl})$ & $P$ Value & OR $(95 \% \mathrm{Cl})$ & $P$ Value \\
\hline Age (yr) & $1.051(1.012-1.092)$ & .010 & $1.050(1.004-1.098)$ & .034 \\
\hline \multicolumn{5}{|l|}{ Sex } \\
\hline Female & Reference group & & & \\
\hline Male & $1.435(0.729-2.823)$ & .296 & $1.561(0.699-3.489)$ & .278 \\
\hline Hypertension (present) & $1.475(0.757-2.875)$ & .254 & & \\
\hline Diabetes (present) & $1.495(0.627-3.564)$ & .364 & & \\
\hline Hyperlipidemia (present) & $1.074(0.416-2.774)$ & .883 & & \\
\hline Previous stroke (present) & 0.470 (0.131-1.681) & .245 & & \\
\hline$A P O E \varepsilon 4$ allele (present) & $3.526(1.630-7.627)$ & .001 & $4.583(1.945-10.796)$ & $<.001$ \\
\hline High degree of MR imaging-visible PVS-CS (score, $\geq 3$ ) & $2.966(1.503-5.851)$ & .002 & $2.307(1.036-5.136)$ & .041 \\
\hline High degree of MR imaging-visible PVS-BG (score, $\geq 3$ ) & $1.034(0.429-2.492)$ & .940 & & \\
\hline WMH (present) & $2.321(1.188-4.533)$ & .014 & & \\
\hline Lacunes (for 1 number higher) & $0.937(0.726-1.209)$ & .618 & & \\
\hline cSS (present) & $5.577(0.654-47.565)$ & .116 & & \\
\hline Lobar CMB (present) & $1.100(0.934-1.295)$ & .253 & & \\
\hline Deep CMB (present) & $0.688(0.428-1.108)$ & .124 & & \\
\hline
\end{tabular}

Note:-CMB indicates cerebral microbleed; CSS, cortical superficial siderosis; MRI-visible PVS-BG, enlarged perivascular space in the basal ganglia; MRI-visible PVS-CS, enlarged perivascular space in the semi ovale. 
$18.6 \%, P=.018$ ), and a higher number of lobar microbleeds (median, 0 [interquartile range, $0-0$ ] versus 0 [interquartile range, $0-1] ; P=.007)$. Other results are presented in the Online Supplemental Data.

\section{DISCUSSION}

In this retrospective study, we hypothesized that MR imagingvisible PVS-CS would be associated with brain amyloid deposition in patients with ADCI. We found that a high degree of MR imaging-visible PVS-CS were independently associated with $\beta$-amyloid accumulation, as assessed by $\left[{ }^{18} \mathrm{~F}\right]$ FBB PET scanning, even after adjusting for previously known clinical risk factors. Our findings support the evidence that MR imaging-visible PVSCS are associated with the amyloid pathology of the brain and could be an indirect imaging marker of amyloid burden in the brains of patients with ADCI.

PVS become visible on MR imaging when enlarged and can be detected in individuals of all ages; however, MR imaging-visible PVS are more frequently found with aging. ${ }^{17,33}$ A recent metaanalysis, including a total of 8395 individuals, showed strong evidence for the association with age and MR imaging-visible PVS in the basal ganglia, as well as in the centrum semiovale. ${ }^{33}$ On the other hand, a greater number of MR imaging-visible PVS can also be associated with various pathologic conditions. Accumulating evidence shows that MR imaging-visible PVS-CS, in particular, are associated with amyloid-associated pathology; several different studies have demonstrated an association with the presence of $\mathrm{AD}$ and MR imaging-visible PVS-CS, ${ }^{7,20,34}$ though the mechanisms of MR imaging-visible PVS-CS remain poorly understood. However, our observation suggests a potential pathophysiologic link between $\mathrm{AD}$ and MR imaging-visible PVS, in that progressive $\beta$-amyloid deposition in the vascular wall or brain cortex may interfere with the perivascular drainage of interstitial fluid and ultimately cause retrograde perivascular space dilation in the white matter. ${ }^{35}$ Consequently, the visibility of MR imaging-visible PVS is increased on MR imaging and appears as spaces with a signal intensity similar to that of CSF, resulting in a high burden of MR imaging-visible PVS. ${ }^{8}$ Consistent with this hypothesis, a postmortem study of AD demonstrated that the degree of white matter PVS on histopathologic examination was positively correlated with cortical $\beta$-amyloid deposition, ${ }^{16}$ which is in line with our observation. Therefore, visible PVS on brain MR imaging may result from abnormal amyloid accumulation in the cortex and cortical/leptomeningeal vessels.

Recently, advances in bioimaging and radiochemistry have enabled the in vivo imaging of $\beta$-amyloid deposits of $\mathrm{AD}$, and this could be beneficial in aiding the early diagnosis of $\mathrm{AD}$, compared with the use of clinical symptoms alone. ${ }^{36-38}$ Furthermore, the use of biomarkers may also identify individuals who could benefit from disease-modifying therapies in $\mathrm{AD}$. On the basis of our results, MR imaging-visible PVS alone cannot be an alternative to an amyloid PET scan; however, MR imaging-visible PVSCS could be a useful indirect marker of amyloid deposition and may also be helpful in determining which patients with cognitive impairment are most likely to benefit from a biomarker test. These approaches may decrease the unnecessary patient burden and the costs of clinical practice and clinical trials.
To date, unlike the evidence for the cerebral amyloid angiopathy population, ${ }^{14,17}$ scarce evidence exists regarding the association between $\mathrm{AD}$ and the in vivo amyloid burden, as assessed with PET. A previous study, with results conflicting with ours, showed that there was no association between MR imaging-visible PVS and amyloid burden in patients with dementia. ${ }^{7}$ However, several considerable differences were found in terms of the study methodology, which may lead to different study outcomes. ${ }^{7}$ First, the aforementioned study included a population with $\mathrm{AD}$ and vascular cognitive impairment, unlike our ADCI population. Because they included a large vascular dementia population, the heterogeneity of the diagnosis and probable higher severity of small-vessel disease in the population may lead to different results between the 2 studies. The amyloid-negative group in the aforementioned study had an inevitably higher WMH volume load, which may result in the misdiagnosis of MR imaging-visible PVS and ultimately interfere with the evaluation of the association between MR imaging-visible PVS and amyloid positivity. Furthermore, the different cutoff values for MR imaging-visible PVS scores may also lead to different study results. Those investigators considered score 2 (11-20 PVS) as a moderate pathologic condition, whereas we considered score 2 as a low degree of MR imaging-visible PVS. We believe that due to the wider area of the centrum semiovale compared with the basal ganglia and consequent higher number of PVS in the centrum semiovale than in basal ganglia, when one focuses on PVS in the centrum semiovale, score 2 should be considered a lower degree of PVS as it has been in other studies. ${ }^{10,12}$

Most interesting, we found that the group negative for $\beta$-amyloid had many different clinical variables that may be associated with a high degree of MR imaging-visible PVS-CS, such as aging, hypertension, and lower cognitive function in addition to $\mathrm{AD}$ pathology, lobar microbleeds, and the presence of WMHs. Therefore, we hypothesized that in patients with ADCI with lower amyloid burden, the presence of an MR imaging-visible PVS may have multifactorial causes, such as arterial stiffness and atrophy, ${ }^{39,40}$ whereas in patients with ADCI and a high amyloid burden, MR imaging-visible PVS are primarily caused by amyloid accumulation. However, this hypothesis needs further validation.

In multivariate analysis, MR imaging-visible PVS-CS, APOE $\varepsilon 4$ allele presence, and older age were significantly related to $\beta$-amyloid PET positivity, as analyzed by the BAPL scoring system. The visual assessment of $\left[{ }^{18} \mathrm{~F}\right]$ FBB PET images has achieved high diagnostic accuracy, with the neuropathology assessments offering good reliability and efficacy. ${ }^{41,42} \mathrm{We}$ believe that using the BAPL scoring system to evaluate $\beta$-amyloid deposition can achieve accurate and reproducible assessments of $\left[{ }^{18} \mathrm{~F}\right]$ FBB PET data. Also, a semi-quantitative analysis by SUVr cutoff classification was performed to obtain a significant result. Bullich et $\mathrm{al}^{31}$ reported a good agreement between florbetaben PET quantification and histopathologic amyloid plaque density (92\% sensitivity and $96 \%$ specificity) and visual read results by experts (percentage agreement $=94 \% \sim 97 \%$ ). They emphasized the robustness of visual analysis performed by expert readers, as well as the additional contribution that optimized relative FBB uptake quantification may have for the detection of $\beta$-amyloid plaques. 
Our study had some limitations. First, it was a retrospective observational study and may have selection bias. Our findings require external validation in larger cohorts. We also did not quantitatively assess the MR imaging-visible PVS burden in both the centrum semiovale and basal ganglia. Therefore, whether MR imaging-visible PVS in both the centrum semiovale and basal ganglia are consistent and good estimators of the whole-PVS load in the brain remains uncertain. Standardized, fully automated, and reliable whole-brain assessment techniques for PVS volume quantification are needed to generalize the results of our study.

\section{CONCLUSIONS}

The findings of this study provide further supporting evidence that MR imaging-visible PVS-CS are a key imaging marker of amyloid pathology when assessed by amyloid PET scans in patients with ADCI. Our findings raise the possibility that MR imaging-visible PVS-CS in patients with ADCI are also a consequence of amyloid deposition in the cortical and vascular amyloid processes.

\section{REFERENCES}

1. Doubal FN, MacLullich AM, Ferguson KJ, et al. Enlarged perivascular spaces on MRI are a feature of cerebral small vessel disease. Stroke 2010;41:450-54 CrossRef Medline

2. Brown R, Benveniste $H$, Black SE, et al. Understanding the role of the perivascular space in cerebral small vessel disease. Cardiovasc Res 2018;114:1462-73 CrossRef Medline

3. Zhu YC, Tzourio C, Soumare A, et al. Severity of dilated VirchowRobin spaces is associated with age, blood pressure, and MRI markers of small vessel disease: a population-based study. Stroke 2010;41:2483-90 CrossRef Medline

4. Weller RO, Hawkes CA, Kalaria RN, et al. White matter changes in dementia: role of impaired drainage of interstitial fluid. Brain Pathol 2015;25:63-78 CrossRef Medline

5. Wardlaw JM, Benveniste H, Nedergaard M, et al. Colleagues from the Fondation Leducq Transatlantic Network of Excellence on the Role of the Perivascular Space in Cerebral Small Vessel Disease. Perivascular spaces in the brain: anatomy, physiology and pathology. Nat Rev Neurol 2020;16:137-53 CrossRef Medline

6. Park YW, Shin NY, Chung SJ, et al. Magnetic resonance imaging-visible perivascular spaces in basal ganglia predict cognitive decline in Parkinson's disease. Mov Disord 2019;34:1672-79 CrossRef Medline

7. Banerjee G, Kim HJ, Fox Z, et al. MRI-visible perivascular space location is associated with Alzheimer's disease independently of amyloid burden. Brain 2017;140:1107-16 CrossRef Medline

8. Smeijer D, Ikram MK, Hilal S. Enlarged perivascular spaces and dementia: a systematic review. J Alzheimers Dis 2019;72:247-56 CrossRef Medline

9. Inglese M, Bomsztyk E, Gonen O, et al. Dilated perivascular spaces: hallmarks of mild traumatic brain injury. AJNR Am J Neuroradiol 2005;26:719-24 Medline

10. Martinez-Ramirez S, Pontes-Neto OM, Dumas AP, et al. Topography of dilated perivascular spaces in subjects from a memory clinic cohort. Neurology 2013;80:1551-56 CrossRef Medline

11. Charidimou A, Meegahage R, Fox Z, et al. Enlarged perivascular spaces as a marker of underlying arteriopathy in intracerebral haemorrhage: a multicentre MRI cohort study. J Neurol Neurosurg Psychiatry 2013;84:624-29 CrossRef Medline

12. Charidimou A, Boulouis G, Pasi M, et al. MRI-visible perivascular spaces in cerebral amyloid angiopathy and hypertensive arteriopathy. Neurology 2017;88:1157-64 CrossRef Medline
13. van Veluw SJ, Biessels GJ, Bouvy WH, et al. Cerebral amyloid angiopathy severity is linked to dilation of juxtacortical perivascular spaces. J Cereb Blood Flow Metab 2016;36:576-80 CrossRef Medline

14. Raposo N, Planton M, Payoux P, et al. Enlarged perivascular spaces and florbetapir uptake in patients with intracerebral hemorrhage. Eur J Nucl Med Mol Imaging 2019;46:2339-47 CrossRef Medline

15. Martinez-Ramirez S, van Rooden S, Charidimou A, et al. Perivascular spaces volume in sporadic and hereditary (Dutch-Type) cerebral amyloid angiopathy. Stroke 2018;49:1913-19 CrossRef Medline

16. Roher AE, Kuo YM, Esh C, et al. Cortical and leptomeningeal cerebrovascular amyloid and white matter pathology in Alzheimer's disease. Mol Med 2003;9:112-22 CrossRef Medline

17. Charidimou A, Hong YT, Jager HR, et al. White matter perivascular spaces on magnetic resonance imaging: marker of cerebrovascular amyloid burden? Stroke 2015;46:1707-09 CrossRef Medline

18. Rowe CC, Ackerman U, Browne W, et al. Imaging of amyloid beta in Alzheimer's disease with 18F-BAY94-9172, a novel PET tracer: proof of mechanism. Lancet Neurol 2008;7:129-35 CrossRef Medline

19. Hawkes CA, Jayakody N, Johnston DA, et al. Failure of perivascular drainage of beta-amyloid in cerebral amyloid angiopathy. Brain Pathol 2014;24:396-403 CrossRef Medline

20. Ramirez J, Berezuk C, McNeely AA, et al. Visible Virchow-Robin spaces on magnetic resonance imaging of Alzheimer's disease patients and normal elderly from the Sunnybrook Dementia Study. J Alzheimers Dis 2014;43:415-24 CrossRef Medline

21. Marcus C, Mena E, Subramaniam RM. Brain PET in the diagnosis of Alzheimer's disease. Clin Nucl Med 2014;39:e413-22; quiz e23-26 CrossRef Medline

22. Murphy MP, LeVine H 3rd. Alzheimer's disease and the amyloidbeta peptide. J Alzheimers Dis 2010;19:311-23 CrossRef Medline

23. McKhann G, Drachman D, Folstein M, et al. Clinical diagnosis of Alzheimer's disease: report of the NINCDS-ADRDA Work Group under the auspices of Department of Health and Human Services Task Force on Alzheimer's Disease. Neurology 1984;34:939-44 CrossRef Medline

24. Petersen RC, Smith GE, Waring SC, et al. Mild cognitive impairment: clinical characterization and outcome. Arch Neurol 1999;56:303-08 CrossRef Medline

25. Ahn HJ, Chin J, Park A, et al. Seoul Neuropsychological Screening Battery-dementia version (SNSB-D): a useful tool for assessing and monitoring cognitive impairments in dementia patients. $J$ Korean Med Sci 2010;25:1071-76 CrossRef Medline

26. Wardlaw JM, Smith EE, Biessels GJ, et al. STandards for ReportIng Vascular changes on nEuroimaging (STRIVE v1). Neuroimaging standards for research into small vessel disease and its contribution to ageing and neurodegeneration. Lancet Neurol 2013;12:82238 CrossRef Medline

27. Maclullich AM, Wardlaw JM, Ferguson KJ, et al. Enlarged perivascular spaces are associated with cognitive function in healthy elderly men. J Neurol Neurosurg Psychiatry 2004;75:1519-23 CrossRef Medline

28. Fazekas F, Chawluk JB, Alavi A, et al. MR signal abnormalities at $\mathbf{1 . 5}$ $T$ in Alzheimer's dementia and normal aging. AJR Am J Roentgenol 1987;149:351-56 CrossRef Medline

29. Barthel H, Gertz HJ, Dresel S, et al. Cerebral amyloid-beta PET with florbetaben $(18 \mathrm{~F})$ in patients with Alzheimer's disease and healthy controls: a multicentre phase $\mathbf{2}$ diagnostic study. Lancet Neurol 2011;10:424-35 CrossRef Medline

30. Lopresti BJ, Klunk WE, Mathis CA, et al. Simplified quantification of Pittsburgh Compound B amyloid imaging PET studies: a comparative analysis. J Nucl Med 2005;46:1959-72 CrossRef Medline

31. Bullich S, Seibyl J, Catafau AM, et al. Optimized classification of (18)F-Florbetaben PET scans as positive and negative using an SUVR quantitative approach and comparison to visual assessment. Neuroimage Clin 2017;15:325-32 CrossRef Medline

32. Duara R, Loewenstein DA, Lizarraga G, et al. Effect of age, ethnicity, sex, cognitive status and APOE genotype on amyloid load and the threshold for amyloid positivity. Neuroimage Clin 2019;22:101800 CrossRef Medline 
33. Francis F, Ballerini L, Wardlaw JM. Perivascular spaces and their associations with risk factors, clinical disorders and neuroimaging features: a systematic review and meta-analysis. Int J Stroke 2019;14:359-71 CrossRef Medline

34. Chen W, Song X, Zhang Y; Alzheimer's Disease Neuroimaging Initiative. Assessment of the Virchow-Robin Spaces in Alzheimer disease, mild cognitive impairment, and normal aging, using high-field MR imaging. AJNR Am J Neuroradiol 2011;32:1490-95 CrossRef Medline

35. Weller RO, Djuanda E, Yow HY, et al. Lymphatic drainage of the brain and the pathophysiology of neurological disease. Acta Neuropathol 2009;117:1-14 CrossRef Medline

36. Palmqvist S, Zetterberg H, Mattsson N, et al. Alzheimer's Disease Neuroimaging Initiative. Detailed comparison of amyloid PET and CSF biomarkers for identifying early Alzheimer disease. Neurology 2015;85:1240-49 CrossRef Medline

37. Buchhave P, Minthon L, Zetterberg H, et al. Cerebrospinal fluid levels of beta-amyloid 1-42, but not of tau, are fully changed already 5 to 10 years before the onset of Alzheimer dementia. Arch Gen Psychiatry 2012;69:98-106 CrossRef Medline
38. Hansson O, Zetterberg H, Buchhave P, et al. Association between CSF biomarkers and incipient Alzheimer's disease in patients with mild cognitive impairment: a follow-up study. Lancet Neurol 2006;5:22834 CrossRef Medline

39. Hughes TM, Craft S, Lopez OL. Review of 'the potential role of arterial stiffness in the pathogenesis of Alzheimer's disease.' Neurodegener Dis Manag 2015;5:121-35 CrossRef Medline

40. Adams HH, Hilal S, Schwingenschuh P, et al. A priori collaboration in population imaging: the Uniform Neuro-Imaging of VirchowRobin Spaces Enlargement Consortium. Alzheimers Dement (Amst) 2015;1:513-20 CrossRef Medline

41. Minoshima S, Drzezga AE, Barthel H, et al. SNMMI Procedure Standard/EANM Practice Guideline for Amyloid PET Imaging of the Brain 1.0. J Nucl Med 2016;57:1316-22 CrossRef Medline

42. Seibyl J, Barthel H, Stephens A, et al. Reliability, reproducibility and efficacy of the $18 \mathrm{~F}$ florbetaben $\beta$-amyloid PET scan visual assessment method as trained via a computer-based instructional tool. $J$ Nucl Med 2013;54(Suppl 2):300 